\title{
ON THE CONVOLUTION ALGEBRA OF BEURLING
}

\author{
GEN-ICHIRÔ SUNOUCH I
}

(Received April 30, 1967)

1. Introduction. Let $f(x)$ be an integrable function with period $2 \pi$ and its Fourier series be

$$
S(f)=\sum_{n=-\infty}^{\infty} c_{n} e^{i n x}
$$

We write $\widehat{A}$ for the class of functions with absolute convergent Fourier series. $\widehat{A}$ is a Banach algebra under usual operations. In this algebra, spectral synthesis is impossible and operating functions are analytic. A. Beurling [1] considered a subclass of $\hat{A}$ such that

$$
A_{0}=\{f \mid A(f)<\infty\}
$$

where

$$
A(f)=\int_{0}^{1} t^{-3 / 2}\left\{\int_{0}^{2 \pi}|f(x+t)-f(x-t)|^{2} d x\right\}^{1 / 2} d t
$$

The algebra $A_{0}$ has remarkable properties, that is to say, that spectral synthesis is possible and the functions which satisfy the Lipschitz condition of order 1 are operating.

In this note, we extend slightly $A(f)$ to

$$
A_{\beta}(f)=\int_{0}^{1} t^{-2+\beta / 2}\left\{\int_{0}^{2 \pi}|f(x+t)-f(x-t)|^{2} d x\right\}^{\beta / 2} d t
$$

for $1 \leqq \beta<2$ and show that $A_{\beta}(f)<\infty$ is equivalent to $B_{\beta}(f)<\infty$ or $C_{\beta}(f)<\infty$, where

$$
B_{\beta}(f)=\sum_{n=1}^{\infty} n^{-\beta / 2}\left\{\sum_{|k|=n+1}^{\infty}\left|c_{k}\right|^{2}\right\}^{\beta / 2}
$$


and

$$
C_{\beta}(f)=\sum_{n=1}^{\infty} n^{-3 \beta / 2}\left\{\sum_{|k|=1}^{n} k^{2}\left|c_{k}\right|^{2}\right\}^{\beta / 2}
$$

Denote by $s_{n}(x)$ the partial sum of $S(f)$, then

$$
B_{\beta}(f)=\sum_{n=1}^{\infty} n^{-\beta / 2}\left\{\int_{0}^{2 \pi}\left|f(x)-s_{n}(x)\right|^{2} d x\right\}^{\beta / 2}
$$

and

$$
C_{\beta}(f)=\sum_{n=1}^{\infty} n^{-3 \beta / 2}\left\{\int_{0}^{2 \pi}\left|s_{n}^{\prime}(x)\right|^{2} d x\right\}^{\beta / 2}
$$

Therefore the above equivalency has an interpretation for approximation theory.

The above equivalence relation throws also lights on papers of Boas [3] and Kinukawa [5] on the absolute convergence of trigonometric series. We discuss this and related problems in the last section.

2. Equivalence relations. We begin with the equivalency of $B_{\beta}(f)<$ a and $C_{\beta}(f)<\infty$.

THEOREM 1. For $1 \leqq \beta<2$, the finiteness of $B_{\beta}(f)$ is equivalent to the finiteness of $C_{\beta}(f)$.

PROOF. By a principle of the condensation test, $B_{\beta}(f)<\infty$ is equivalent to the finiteness of

$$
B_{\beta}^{\prime}(f)=\sum_{k=0}^{\infty} 2^{k(1-\beta / 2)}\left\{\sum_{|v|=2^{k}+1}^{\infty}\left|c_{\nu}\right|^{2}\right\}^{\beta / 2}
$$

and $C_{\beta}(f)<\infty$ is equivalent to the finiteness of

$$
C_{\beta}^{\prime}(f)=\sum_{k=0}^{\infty} 2^{k(1-3 \beta / 2)}\left\{\sum_{|\nu|=1}^{2^{k+1}} \nu^{2}\left|c_{\nu}\right|^{2}\right\}^{\beta / 2}
$$

Concerning $C_{\beta}^{\prime}(f)$, we have

$$
C_{\beta}^{\prime}(f)=\sum_{k=0}^{\infty} 2^{k(1-3 \beta / 2)}\left\{\sum_{|v|=1}^{2^{k+1}} \nu^{2}\left|c_{\nu}\right|^{2}\right\}^{\beta / 2}
$$




$$
\begin{aligned}
& =\sum_{k=0}^{\infty} 2^{k(1-3 \beta / 2)}\left\{\sum_{m=0}^{k} \sum_{|\nu|=2^{m}+1}^{2^{m+1}} \nu^{2}\left|c_{v}\right|^{2}\right\}^{\beta / 2} \\
& \leqq \sum_{k=0}^{\infty} 2^{k(1-3 \beta / 2)}\left\{4 \sum_{m=0}^{k} 2^{2 m} \sum_{|\nu|=2^{m}+1}^{2^{m+1}}\left|c_{\nu}\right|^{2}\right\}^{\beta / 2} .
\end{aligned}
$$

Since $\beta / 2<1$, by Jensen's inequality, the last term is less than

$$
\begin{aligned}
K \sum_{k=0}^{\infty} 2^{k(1-3 \beta / 2)} & \left\{\sum_{m=0}^{k} 2^{m \beta}\left(\sum_{|v|=2^{m}+1}^{2^{m+1}}\left|c_{\nu}\right|^{2}\right)^{\beta / 2}\right\} \\
& =K \sum_{m=0}^{\infty} 2^{m \beta}\left(\sum_{|\nu|=2^{m}+1}^{2^{m+1}}\left|c_{\nu}\right|^{2}\right)^{\beta / 2} \sum_{k=m}^{\infty} 2^{k(1-3 \beta / 2)} \\
& =K \sum_{m=0}^{\infty} 2^{m(1-\beta / 2)}\left\{\sum_{\mid=2^{m}+1}^{2^{m+1}}\left|c_{\nu}\right|^{2}\right\}^{\beta / 2} \\
& \leqq K \sum_{m=0}^{\infty} 2^{m(1-\beta / 2)}\left\{\sum_{|\nu|=2^{m}+1}^{\infty}\left|c_{\nu}\right|^{2}\right\}^{\beta / 2} \\
& =K B_{\beta}^{\prime}(f) .
\end{aligned}
$$

Concerning the converse part, we proceed with the same method.

$$
\begin{aligned}
B_{\beta}^{\prime}(f) & =\sum_{k=0}^{\infty} 2^{k(1-\beta / 2)}\left\{\sum_{|\nu|=2^{k+1}}^{\infty}\left|c_{\nu}\right|^{2}\right\}^{\beta / 2} \\
& =\sum_{k=0}^{\infty} 2^{k(1-\beta / 2)}\left\{\sum_{m=k}^{\infty} \sum_{|\nu|=2^{m}+1}^{2^{m+1}}\left|c_{\nu}\right|^{2}\right\}^{\beta / 2} \\
& \leqq \sum_{k=0}^{\infty} 2^{k(1-\beta / 2)}\left\{\sum_{m=k}^{\infty} 2^{-2 m}\left(\sum_{|\nu|=2^{m}+1}^{2^{m+1}} \nu^{2}\left|c_{\nu}\right|^{2}\right)\right\}^{\beta / 2}
\end{aligned}
$$

By Jensen's inequality, we have

$$
\begin{aligned}
& \leqq \sum_{k=0}^{\infty} 2^{k(1-\beta / 2)}\left\{\sum_{m=k}^{\infty} 2^{-m \beta}\left(\sum_{|\nu|=2^{m}+1}^{2^{m+1}} \nu^{2}\left|c_{\nu}\right|^{2}\right)^{\beta / 2}\right\} \\
& =\sum_{m=0}^{\infty} 2^{-m \beta}\left(\sum_{|\nu|=2^{m}+1} \nu^{2}\left|c_{\nu}\right|^{2}\right)^{\beta / 2} \sum_{k=0}^{m} 2^{k(1-\beta / 2)} \\
& \leqq K \sum_{m=0}^{\infty} 2^{m(1-3 \beta / 2)}\left\{\sum_{|\nu|=1}^{2^{m+1}} \nu^{2}\left|c_{\nu}\right|^{2}\right\}^{\beta / 2} \\
& =K C_{\beta}^{\prime}(f) .
\end{aligned}
$$


THEOREM 2. For $1 \leqq \beta<2$, the finiteness of $A_{\beta}(f)$ is equivalent to the finiteness of $B_{\beta}(f)$ or $C_{\beta}(f)$.

PROOF. Since

$$
\int_{0}^{2 \pi}|f(x+t)-f(x-t)|^{2} d x=4 \pi \sum_{k=-\infty}^{\infty}\left|c_{k}\right|^{2} \sin ^{2} k t
$$

we have

$$
\begin{aligned}
A_{\beta}(f) & =\int_{0}^{1} t^{-2+\beta / 2}\left\{\int_{0}^{2 \pi}|f(x+t)-f(x-t)|^{2} d x\right\}^{\beta / 2} d t \\
& =K \int_{0}^{1} t^{-2+\beta / 2}\left\{\sum_{|k|=1}^{\infty}\left|c_{k}\right|^{2} \sin ^{2} k t\right\}^{\beta / 2} d t \\
& \leqq K \sum_{n=2}^{\infty} \int_{n^{-1}}^{(n-1)^{-1}} t^{-2+\beta / 2}\left\{\left(\sum_{|k|=1}^{n}\left|c_{k}\right|^{2} k^{2} t^{2}\right)^{\beta / 2}+\left(\sum_{k=n+1}^{\infty}\left|c_{k}\right|^{2}\right)^{\beta / 2}\right\} d t \\
& \leqq K \sum_{n=2}^{\infty}\left(\sum_{|k|=1}^{n} k^{2}\left|c_{k}\right|^{2}\right)^{\beta / 2} \int_{n^{-1}}^{(n-1)^{-1}} t^{-2+3 \beta / 2} d t+K \sum_{n=2}^{\infty}\left(\sum_{|k|=n+1}^{\infty}\left|c_{k}\right|^{2}\right)^{\beta / 2} \int_{n^{-1}}^{(n-1)^{-1}} t^{-2+\beta / 2} d t \\
& \leqq K \sum_{n=2}^{\infty}\left(\sum_{k=1}^{n}\left|c_{k}\right|^{2} k^{2}\right)^{\beta / 2} n^{-3 \beta / 2}+K \sum_{n=2}^{\infty}\left(\sum_{k=n+1}^{\infty}\left|c_{k}\right|^{2}\right)^{\beta / 2} n^{-\beta / 2} \\
& \leqq K C_{\beta}(f)+K B_{\beta}(f) .
\end{aligned}
$$

On the other hand,

$$
\begin{aligned}
A_{\beta}(f)=\int_{0}^{1} t^{-2+\beta / 2} & \left\{\int_{0}^{2 \pi}|f(x+t)-f(x-t)|^{2} d x\right\}^{\beta / 2} d t \\
& =K \int^{1} t^{-2+\beta / 2}\left\{\sum_{|k|=1}^{\infty}\left|c_{k}\right|^{2} \sin ^{2} k t\right\}^{\beta / 2} d t \\
& \geqq K \sum_{n=2}^{\infty} \int_{n^{-1}}^{(n-1)^{-1}} t^{-2+\beta / 2}\left\{\sum_{k=1}^{n}\left|c_{k}\right|^{2} \sin ^{2} k t\right\}^{\beta / 2} d t
\end{aligned}
$$

When $k t \leqq 1$, we have $\sin k t \geqq A k t$, and the last term is greater than

$$
K \sum_{n=2}^{\infty} \int_{n^{-1}}^{(n-1)^{-1}} t^{-2+3 \beta / 2}\left\{\sum_{|k|=1}^{n} k^{2}\left|c_{k}\right|^{2}\right\}^{\beta / 2} d t \geqq K \sum_{n=2}^{\infty} n^{-3 \beta / 2}\left\{\sum_{|k|=1}^{n} k^{2}\left|c_{k}\right|^{2}\right\}^{\beta / 2} .
$$


Thus the theorem is proved.

The equivalency of $A_{\beta}(f)<\infty$ to $B_{\beta}(f)<\infty$ is proved by Leindler [9] already and M. and S. Izumi [4] gave a simple proof for $A_{\beta}(f) \geqq K B_{\beta}(f)$, also.

3. Convergence of $\sum\left|c_{n}\right|^{\beta}$ and related problems. Stechkin proved very simply that $B(f)<\infty$ implies the absolute convergence of $\sum c_{n}$. The same proof gives the following theorem.

THEOREM 3. If $B_{\beta}(f)<\infty$ for $1 \leqq \beta<2$, then $\sum\left|c_{n}\right|^{\beta}<\infty$.

ProOF. Without loss of generality, we can suppose that $f(x)$ is even. By Hölder's inequality,

$$
\begin{aligned}
\sum_{k=1}^{\infty}\left|c_{k}\right|^{\beta} & =\sum_{k=1}^{\infty} \sum_{n=1}^{k} \frac{\left|c_{k}\right|^{\beta}}{k} \\
& =\sum_{n=1}^{\infty} \sum_{k=n}^{\infty} \frac{\left|c_{k}\right|^{\beta}}{k} \\
& \leqq \sum_{n=1}^{\infty}\left\{\sum_{k=n}^{\infty} k^{-2 /(2-\beta)}\right\}^{(2-\beta) ! 2}\left\{\sum_{k=n}^{\infty}\left(\left|c_{k}\right|^{\beta}\right)^{2 / \beta}\right\}^{\beta / 2} \\
& \leqq \sum_{n=1}^{\infty} n^{-\beta / 2}\left(\sum_{k=n}^{\infty}\left|c_{k}\right|^{2}\right)^{\beta / 2} \\
& =B_{\beta}(f)<\infty .
\end{aligned}
$$

THEOREM 4. If $f(x)$ and $g(x)$ are continuous even function, of period $2 \pi$, with Fouricr coefficient $c_{n}$ and $d_{n}$, if $f(x)$ is a contraction of $g(x)$, and if $\left|d_{n}\right| \leqq \gamma_{n}$ where

$$
\sum_{n=1}^{\infty} n^{-3 \beta / 2}\left\{\sum_{k=1}^{n} k^{2} \gamma_{k}^{2}\right\}^{\beta / 2}<\infty
$$

or

$$
\sum_{n=1}^{\infty} n^{-\beta / 2}\left\{\sum_{k=n+1}^{\infty} \gamma_{k}^{2}\right\}^{\beta / 2}<\infty
$$

then $\sum\left|c_{n}\right|^{\beta}<\infty$. 
PROOF. That $f(x)$ is a contraction of $g(x)$ means

$$
|f(x)-f(y)| \leqq|g(x)-g(y)|
$$

Since (4) or (5) is equivalent to $A_{\beta}(f)<\infty$, Theorem is immediate from Theorem 3 and the form of $A_{\beta}(f)$.

Boas [3] and Kinukawa [5] proved Theorem 4 under the conditions (4) and (5).

COROLLARY. Theorem 4 remains true when the hypothesis (4) or (5) is replaced by $\gamma_{n} \downarrow 0$ and $\sum\left|\gamma_{n}\right|^{\beta}<\infty$.

Boas [3] and Konyushkov [7] proved that the hypothesis of corollary implies (4) and (5) and, equivalent to $A_{\beta}(f)<\infty$ by Theorem 2 .

Kinukawa $[6,7]$ also discussed the problem of spectral synthesis under the conditions (4) and (5). However it is sufficient under (4) or (5) and this turns to $A_{\beta}(f)<\infty$ and reduces to Beurling's idea.

In particular, when $\beta=1$, as Beurling shows,

$$
\left.A_{0}=\{f \mid A(f)<\infty\} *\right)
$$

is an algebra. In fact, we have

$$
\begin{aligned}
A(f g)= & \int_{0}^{1} t^{-3 / 2}\left\{\int_{0}^{2 \pi}|f(x+t) g(x+t)-f(x-t) g(x-t)|^{2} d x\right\}^{1 / 2} d t \\
& \leqq \int_{0}^{1} t^{-3 / 2}\left\{\int_{0}^{2 \pi} \mid f(x+t) g(x+t)-f(x-t) g(x+t)\right. \\
& \left.\quad+f(x-t) g(x+t)-\left.f(x-t) g(x-t)\right|^{2} d x\right\}^{1 / 2} d t \\
& \leqq \max _{0 \leqq x \leqq 2 \pi}|g(x)| A(f)+\max _{0 \leqq x \leqq 2 \pi}|f(x)| A(g) \\
\leqq &
\end{aligned}
$$

because

$$
\max _{0 \leqq a \leqq 2 \pi}|f(x)| \leqq \sum_{n=-\infty}^{\infty}\left|c_{n}\right| \leqq B(f) \leqq A(f)
$$

The equivalency of $A(f)<\infty, B(f)<\infty$ and $C(f)<\infty$ gives the following inequalities between the formal products of Fourier coefficients.

*) $A(f)$ means $A_{\beta}(f)$ when $\beta=1$ and $B(f), C(f)$ are the same. 
Let

$$
\begin{aligned}
& f(x) \sim \sum_{n=-\infty}^{\infty} c_{n} e^{i n x} \\
& g(x) \sim \sum_{n=-\infty}^{\infty} d_{n} e^{i n x}
\end{aligned}
$$

and

$$
f(x) g(x) \sim \sum_{n=-\infty}^{\infty} \gamma_{n} e^{i n x}
$$

where

$$
\gamma_{n}=\sum_{k=-\infty}^{\infty} c_{k} d_{n-k}
$$

THEOREM 5. We have the following inequalities

$$
\begin{aligned}
\sum_{n=1}^{\infty} n^{-1 / 2} & \left(\sum_{|k|=n+1}^{\infty}\left|\gamma_{k}\right|^{2}\right)^{1 / 2} \\
& \leqq K\left\{\sum_{n=1}^{\infty} n^{-1 / 2}\left(\sum_{|k|=n+1}^{\infty}\left|c_{k}\right|^{2}\right)^{1 / 2}\right\}\left\{\sum_{n=1}^{\infty} n^{-1 / 2}\left(\sum_{|k|=n+1}^{\infty}\left|d_{k}\right|^{2}\right)^{1 / 2}\right\}
\end{aligned}
$$

and

$$
\begin{aligned}
\sum_{n=1}^{\infty} n^{-3 / 2} & \left(\sum_{|k|=1}^{n} k^{2}\left|\gamma_{k}\right|^{2}\right)^{1 / 2} \\
& \leqq K\left\{\sum_{n=1}^{\infty} n^{-3 / 2}\left(\sum_{|k|=1}^{n} k^{2}\left|c_{k}\right|^{2}\right)^{1 / 2}\right\}\left\{\sum_{n=1}^{\infty} n^{-3 / 2}\left(\sum_{|k|=1}^{n} k^{2}\left|d_{k}\right|^{2}\right)^{1 / 2}\right\}
\end{aligned}
$$

where $K$ is a constant.

REMARK. Actually Beurling considers Fourier transforms. In Fourier integral case, $f(x)$ does not necessarily belong to the class $L^{2}(-\infty, \infty)$, but only

$$
\int_{0}^{x} t^{2}\left\{|f(t)|^{2}+|f(-t)|^{2}\right\} d t<\infty, \quad \int_{x}^{\infty}\left\{|f(t)|^{2}+|f(-t)|^{2}\right\} d t<\infty
$$

for any fixed positive $x$. Hence calculation is somewhat troublesome, but we get analogous propositions. 


\section{REFERENCES}

[1] A. BEURI.ING, Contraction and analysis of some convolution algebras, Ann. Inst. Fourier, 14(1964), 1-32.

[2] R. P. BoAs, Beurling's test for absolute convergence of Fourier series, Bull. Amer. Math. Soc., 66(1960), 24-27.

[3] R. P. BOAS, Inequalities for monotonic series, Journ. Math. Analysis and Aplication, 1(1960), 121-126.

[4] M. IZUMI AND S. IZUMI, On the Leindler theorem, Proc. Japan Acad., 42(1966), 533534.

[5] M. KINUKAwA, Contractions of Fourier coefficients and Fourier integrals, Journ. d'Analyse Math., 8(1960), 377-406.

[6] M. KINUKAWA, On the spectral synthesis of bounded functions, Proc. Amer. Math. Soc., 14(1963), 468-471.

[7] M. KinUkAWA, A note on the closure of translations in $L^{p}$, Tôhoku Math. Journ., 18(1966), 223-235.

[8] A. A. KONYUSHKOV, Best approximation by trigonometric polynomials and Fourier coefficients, Math. Sbornik, 86(1958), 53-84.

[9] L. LEINDLER, Über Strukturbedingungen für Fourierreihen, Math. Zeitschr., 88(1965), $418-431$.

MATHEMATICAL INSTITUTE

TÔHOKU UNIVERSITY,

SENDAI, JAPAN. 